\title{
TRACE ANOMALY AND QUANTIZATION OF MAXWELL'S THEORY ON NON-COMMUTATIVE SPACES
}

\author{
S.I. Kruglov \\ International Educational Centre, 2727 Steeles Ave. W, \# 202, \\ Toronto, Ontario, Canada M3J $3 G 9$
}

\begin{abstract}
The canonical and symmetrical energy-momentum tensors and their non-zero traces in Maxwell's theory on non-commutative spaces have been found. Dirac's quantization of the theory under consideration has been performed. I have found the extended Hamiltonian and equations of motion in the general gauge covariant form.
\end{abstract}

\section{Introduction}

Non-commutative (NC) gauge theories are of interest now because they appear in the superstring theory [1]. In the presence of the external background magnetic field, NC coordinates emerge naturally [1]. The gauge theories on $\mathrm{NC}$ spaces are equivalent (the Seiberg-Witten map) to effective commutative theories with the additional deformation parameters $\theta_{\mu \nu}$. This allows us to formulate a Lagrange field theory on $\mathrm{NC}$ spaces with the same degrees of freedom in terms of ordinary fields. The effective Lagrangian is formulated in terms of ordinary fields and the parameters $\theta_{\mu \nu}$. The coordinates of $\mathrm{NC}$ spaces obey the commutation relation [2], [3]: $\left[\widehat{x}_{\mu}, \widehat{x}_{\nu}\right]=i \theta_{\mu \nu}$. We use here the Lorentz-Heaviside units, and set $\hbar=c=1$.

The effective Lagrangian density in terms of ordinary fields, which is equivalent to Maxwell's Lagrangian density on $\mathrm{NC}$ spaces, in the leading order of $\theta$ is [4]

$$
\mathcal{L}=-\frac{1}{4} F_{\mu \nu}^{2}+\frac{1}{8} \theta_{\alpha \beta} F_{\alpha \beta} F_{\mu \nu}^{2}-\frac{1}{2} \theta_{\alpha \beta} F_{\mu \alpha} F_{\nu \beta} F_{\mu \nu}+\mathcal{O}\left(\theta^{2}\right) .
$$

The field strength tensor is given by

$$
F_{\mu \nu}=\partial_{\mu} A_{\nu}-\partial_{\nu} A_{\mu},
$$

where $A_{\mu}=\left(\mathbf{A}, i A_{0}\right)$ is the electromagnetic field vector-potential; $E_{i}=i F_{i 4}$ is the electric field; $B_{i}=\epsilon_{i j k} F_{j k}\left(\epsilon_{123}=1\right)$ is the magnetic induction field. 
The BRST-shift symmetry and the vacuum polarization of photons at the one-loop level of the theory under consideration have been investigated in [5]. Authors of [6], [7] showed that the velocity of photon propagation in the direction that is perpendicular to a background magnetic induction field in such a $\theta$-deformed Maxwell theory differs from $c$. The Lagrangian (1) can also be rewritten as follows:

$$
\mathcal{L}=\frac{1}{2}\left(\mathbf{E}^{2}-\mathbf{B}^{2}\right)[1+(\theta \cdot \mathbf{B})]-(\theta \cdot \mathbf{E})(\mathbf{E} \cdot \mathbf{B})+\mathcal{O}\left(\theta^{2}\right),
$$

where $\theta_{i}=(1 / 2) \epsilon_{i j k} \theta_{j k}$, and we put $\theta_{i 4}=0$ which guarantees the unitarity [8], [9]. Parameters $\theta_{\mu \nu}$ are the $\mathrm{CP}$ violating variables (at CP transformations $\left.\theta_{\mu \nu} \rightarrow-\theta_{\mu \nu}\right)$, and, as a result, particles possess the dipole moments [10], [11]. Quantum electrodynamics on NC spaces is a renormalizable theory [12], [4] with the conservation of CPT symmetry.

From Eq. (1) equations of motion [6], [7] read

$$
\frac{\partial}{\partial t} \mathbf{D}-\operatorname{rot} \mathbf{H}=0, \quad \operatorname{div} \mathbf{D}=0
$$

where $(\operatorname{rot} \mathbf{H})_{i}=\epsilon_{i j k} \partial_{j} H_{k}$ and $\operatorname{div} \mathbf{D}=\partial_{i} D_{i}$. The displacement $(\mathbf{D})$ and magnetic $(\mathbf{H})$ fields are given by

$$
\begin{gathered}
\mathbf{D}=\mathbf{E}+\mathbf{d}, \quad \mathbf{d}=(\theta \cdot \mathbf{B}) \mathbf{E}-(\theta \cdot \mathbf{E}) \mathbf{B}-(\mathbf{E} \cdot \mathbf{B}) \theta, \\
\mathbf{H}=\mathbf{B}+\mathbf{h}, \quad \mathbf{h}=(\theta \cdot \mathbf{B}) \mathbf{B}+(\theta \cdot \mathbf{E}) \mathbf{E}-\frac{1}{2}\left(\mathbf{E}^{2}-\mathbf{B}^{2}\right) \theta .
\end{gathered}
$$

Eq. (2) leads to $\partial_{\mu} \widetilde{F}_{\mu \nu}=0$ (where $\widetilde{F}_{\mu \nu}=(1 / 2) \varepsilon_{\mu \nu \alpha \beta} F_{\alpha \beta}, \varepsilon_{\mu \nu \alpha \beta}$ is an antisymmetric tensor, $\left.\varepsilon_{1234}=-i\right)$ which is rewritten as

$$
\frac{\partial}{\partial t} \mathbf{B}+\operatorname{rot} \mathbf{E}=0, \quad \operatorname{div} \mathbf{B}=0
$$

\section{Energy-momentum Tensor}

Using the general procedure, we obtain the canonical conservative energymomentum tensor [13]:

$$
T_{\mu \nu}=-F_{\mu \alpha} F_{\nu \alpha}\left(1-\frac{1}{2} \theta_{\gamma \beta} F_{\gamma \beta}\right)+\frac{1}{4} \theta_{\mu \alpha} F_{\nu \alpha} F_{\rho \beta}^{2}
$$




$$
-\theta_{\mu \beta} F_{\gamma \nu} F_{\rho \beta} F_{\gamma \rho}-\left(F_{\mu \alpha} F_{\nu \gamma}+F_{\nu \alpha} F_{\mu \gamma}\right) \theta_{\alpha \beta} F_{\gamma \beta}-\delta_{\mu \nu} \mathcal{L} .
$$

The canonical energy-momentum tensor (7) is non-symmetric, but for classical electrodynamics at $\theta \rightarrow 0$, it becomes symmetric. The trace of the energy-momentum tensor (7) is not equal to zero; that indicates the trace anomaly at the classical level. This is the consequence of the violation of the dilatation symmetry: $x_{\mu}^{\prime}=\lambda x_{\mu}$ for $\mathrm{NC}$ spaces.

Using the general procedure [14], and varying the action corresponding to the Lagrangian (1) on the metric tensor, we find the symmetric energymomentum tensor:

$$
T_{\mu \nu}^{s y m}=T_{\mu \nu}+\frac{1}{4} \theta_{\nu \alpha} F_{\mu \alpha} F_{\rho \beta}^{2}-\theta_{\nu \beta} F_{\gamma \mu} F_{\rho \beta} F_{\gamma \rho} .
$$

Here the conservative tensor $T_{\mu \nu}$ is defined by Eq. (7). The components of the stress tensor tensor, found from Eq. (8), are given by

$$
\begin{gathered}
T_{44}^{s y m}=\frac{\mathbf{E}^{2}+\mathbf{B}^{2}}{2}[1+(\theta \cdot \mathbf{B})]-(\mathbf{E} \cdot \mathbf{B})(\theta \cdot \mathbf{E}) . \\
T_{m 4}^{s y m}=-i\left\{[1+(\theta \cdot \mathbf{B})](\mathbf{E} \times \mathbf{B})+\frac{1}{2}\left(\mathbf{B}^{2}-\mathbf{E}^{2}\right)(\mathbf{E} \times \theta)\right\}_{m} . \\
T_{m n}^{s y m}=E_{m} E_{n}+B_{m} B_{n}-\frac{1}{2} \delta_{m n}\left(\mathbf{E}^{2}+\mathbf{B}^{2}\right)+(\theta \cdot \mathbf{B})\left(3 E_{m} E_{n}+B_{m} B_{n}\right) \\
+\frac{1}{2}\left(\mathbf{E}^{2}+\mathbf{B}^{2}\right)\left[B_{m} \theta_{n}+\theta_{m} B_{n}-3 \delta_{m n}(\theta \cdot \mathbf{B})\right] \\
-(\mathbf{E} \cdot \mathbf{B})\left[\theta_{m} E_{n}+\theta_{n} E_{m}-\delta_{m n}(\theta \cdot \mathbf{E})\right]-(\theta \cdot \mathbf{E})\left(E_{m} B_{n}+E_{n} B_{m}\right) \\
-(\mathbf{E} \times \theta)_{m}(\mathbf{B} \times \mathbf{E})_{n}-(\mathbf{E} \times \theta)_{n}(\mathbf{B} \times \mathbf{E})_{m} .
\end{gathered}
$$

Eqs. (9)-(11) lead to the trace (anomaly) of the symmetric energy-momentum tensor:

$$
T_{\mu \mu}^{s y m}=2(\theta \cdot \mathbf{B})\left(\mathbf{E}^{2}-\mathbf{B}^{2}\right)-4(\theta \cdot \mathbf{E})(\mathbf{E} \cdot \mathbf{B}) .
$$

From Eqs. (7), (12), we find the relation $T_{\mu \mu}^{s y m}=2 T_{\mu \mu}$. The trace anomaly can contribute to the cosmological constant, and as a result, this trace anomaly should be taken into account by consideration of the inflation theory.

The trace anomaly vanishes in the case of the plane electromagnetic waves as two Lorentz invariants $\mathbf{E}^{2}-\mathbf{B}^{2},(\mathbf{E} \cdot \mathbf{B})$ are equal to zero, as well as for classical electrodynamics at $\theta=0$. 


\section{Dirac's Quantization}

In accordance with the general procedure [15] of gauge covariant quantization, we find from Eq. (1) (in leading order of $\theta$ )) the momenta:

$$
\begin{gathered}
\pi_{i}=\frac{\partial \mathcal{L}}{\partial\left(\partial_{0} A_{i}\right)}=-E_{i}[1+(\theta \cdot \mathbf{B})]+(\theta \cdot \mathbf{E}) B_{i}+(\mathbf{E} \cdot \mathbf{B}) \theta_{i}, \\
\pi_{0}=\frac{\partial \mathcal{L}}{\partial\left(\partial_{0} A_{0}\right)}=0
\end{gathered}
$$

A primary constraint follows from Eq. (13):

$$
\varphi_{1}(x)=\pi_{0}, \quad \varphi_{1}(x) \approx 0 .
$$

Here Dirac's symbol $\approx$ is used for equations which hold only weakly. Taking into account the equality $\pi_{i}=-D_{i}$, we find the Poisson brackets

$$
\begin{aligned}
\left\{A_{i}(\mathbf{x}, t), D_{j}(\mathbf{y}, t)\right\} & =-\delta_{i j} \delta(\mathbf{x}-\mathbf{y}), \\
\left\{B_{i}(\mathbf{x}, t), D_{j}(\mathbf{y}, t)\right\} & =\epsilon_{i j k} \partial_{k} \delta(\mathbf{x}-\mathbf{y}) .
\end{aligned}
$$

In the quantum theory, we should make the replacement $\{B, D\} \rightarrow-i[B, D]$, where $[B, D]=B D-D B$ is the commutator. Using Eqs. (3), (13), and the relation $\mathcal{H}=\pi_{\mu} \partial_{0} A_{\mu}-\mathcal{L}$, we arrive at the density of the Hamiltonian:

$$
\mathcal{H}=\frac{1}{2}\left(\mathbf{E}^{2}+\mathbf{B}^{2}\right)[1+(\theta \cdot \mathbf{B})]-(\theta \cdot \mathbf{E})(\mathbf{E} \cdot \mathbf{B})-\pi_{m} \partial_{m} A_{0}
$$

As the primary constraint (14) have to be a constant of motion, we find the condition

$$
\partial_{0} \pi_{0}=\left\{\pi_{0}, H\right\}=-\partial_{m} \pi_{m}=0,
$$

where $H=\int d^{3} x \mathcal{H}$ is the Hamiltonian. Eq. (18) leads to the secondary constraint:

$$
\varphi_{2}(x)=\partial_{m} \pi_{m}, \quad \varphi_{2}(x) \approx 0 .
$$

This is simply the Gauss law (see Eq. (4)). It is easy to find $\partial_{0} \varphi_{2}=$ $\left\{\varphi_{2}, H\right\} \equiv 0$, i.e. no additional constraints. There are no second class constrains here because $\left\{\varphi_{1}, \varphi_{2}\right\}=0$, as similar to classical electrodynamics [15]. In accordance with the general approach [15], to have the total density of Hamiltonian, we add to Eq. (17) the Lagrange multiplier terms:

$$
\mathcal{H}_{T}=\mathcal{H}+v(x) \pi_{0}+u(x) \partial_{m} \pi_{m}
$$


The density energy, obtained from Eq. (17), is $\mathcal{E} \equiv T_{44}^{\text {sym }}$ (see Eq. (9)). The electric field, with the accuracy of $\mathcal{O}\left(\theta^{2}\right)$, is given by

$$
E_{i}=-\pi_{i}[1-(\theta \cdot \mathbf{B})]-(\theta \cdot \pi) B_{i}-(\pi \cdot \mathbf{B}) \theta_{i},
$$

so that the total density of Hamiltonian becomes

$$
\begin{gathered}
\mathcal{H}_{T}=\frac{\pi^{2}+\mathbf{B}^{2}}{2}+(\theta \cdot \mathbf{B}) \frac{\mathbf{B}^{2}-\pi^{2}}{2}+(\theta \cdot \pi)(\pi \cdot \mathbf{B}) \\
+v(x) \pi_{0}+\left(u(x)+A_{0}\right) \partial_{m} \pi_{m},
\end{gathered}
$$

where $\mathbf{B}=\operatorname{rot} \mathbf{A}$.

Using Eq. (22), we obtain the Hamiltonian equations

$$
\begin{gathered}
\partial_{0} A_{i}=\left\{A_{i}, H\right\}=\frac{\delta H}{\delta \pi_{i}} \\
=\pi_{i}[1-(\theta \cdot \mathbf{B})]+(\mathbf{B} \cdot \pi) \theta_{i}+(\pi \cdot \theta) B_{i}-\partial_{i} A_{0}-\partial_{i} u(x), \\
\partial_{0} \pi_{i}=\left\{\pi_{i}, H\right\}=-\frac{\delta H}{\delta A_{i}}=\partial_{n}\left\{\left[\left(\partial_{n} A_{i}\right)-\left(\partial_{i} A_{n}\right)\right][1+(\theta \cdot \mathbf{B})]\right\} \\
+\varepsilon_{i a b} \partial_{b}\left[\theta_{a} \frac{\mathbf{B}^{2}-\pi^{2}}{2}+\pi_{a}(\pi \cdot \theta)\right], \\
\partial_{0} A_{0}=\left\{A_{0}, H\right\}=\frac{\delta H}{\delta \pi_{0}}=v(x), \partial_{0} \pi_{0}=\left\{\pi_{0}, H\right\}=-\frac{\delta H}{\delta A_{0}}=-\partial_{m} \pi_{m},
\end{gathered}
$$

which are gauge-equivalent to the Euler-Lagrange equations. Taking into account the definition (5), we find that Eq. (24) coincides with the first equation in (4), and Eq. (23) is the gauge covariant form of Eq. (21). Gauss's law is the secondary constraint in this Hamiltonian formalism and is the second equation in (4). The first class constraints generate gauge transformations. In accordance with Eq. (25), the $A_{0}$ is arbitrary function and Eqs. (25) represent the time evolution of non-physical fields. The variables $\pi_{0}, \partial_{m} \pi_{m}$ equal zero as constraints. 


\section{The Coulomb Gauge}

Using the gauge freedom here the radiation gauge will be considered. It should be noted that the gauge fixing approach is beyond the Dirac's approach. Using the gauge freedom (described by functions $v(x), u(x)$ ), we impose new constraints:

$$
\varphi_{3}(x)=A_{0} \approx 0, \quad \varphi_{4}(x)=\partial_{m} A_{m} \approx 0 .
$$

Then equations (see also [16])

$$
\left\{\varphi_{1}(\mathbf{x}, t), \varphi_{3}(\mathbf{y}, t)\right\}=-\delta(\mathbf{x}-\mathbf{y}), \quad\left\{\varphi_{2}(\mathbf{x}, t), \varphi_{4}(\mathbf{y}, t)\right\}=\Delta_{x} \delta(\mathbf{x}-\mathbf{y}),
$$

hold, where $\Delta_{x} \equiv \partial^{2} /\left(\partial x_{m}\right)^{2}$. Defining "coordinates" $Q_{i}$ and conjugated momenta $P_{i}(i=1,2)$ :

$$
Q_{i}=\left(A_{0}, \partial_{m} A_{m}\right), \quad P_{i}=\left(\pi_{0},-\Delta_{x}^{-1} \partial_{m} \pi_{m}\right),
$$

we find

$$
\left\{Q_{i}(\mathbf{x}, t), P_{j}(\mathbf{y}, t)\right\}=\delta_{i j} \delta(\mathbf{x}-\mathbf{y}), \quad \Delta_{x}^{-1}=-\frac{1}{4 \pi|\mathbf{x}|}, \quad \Delta_{x} \frac{1}{4 \pi|\mathbf{x}|}=-\delta(\mathbf{x}),
$$

Canonical variables $Q_{i}, P_{i}$, Eq. (29) are not the physical degrees of freedom and must be eliminated. Using the matrix of Poisson brackets [16] $C_{i j}=$ $\left\{\varphi_{i}(\mathbf{x}, t), \varphi_{j}(\mathbf{y}, t)\right\}$, and the definition of the Dirac brackets [16], [17], we obtain

$$
\begin{gathered}
\left\{\pi_{0}(\mathbf{x}, t), A_{0}(\mathbf{y}, t)\right\}^{*}=\left\{\pi_{0}(\mathbf{x}, t), A_{i}(\mathbf{y}, t)\right\}^{*}=\left\{\pi_{i}(\mathbf{x}, t), A_{0}(\mathbf{y}, t)\right\}^{*}=0 \\
\left\{\pi_{i}(\mathbf{x}, t), A_{j}(\mathbf{y}, t)\right\}^{*}=-\delta_{i j} \delta(\mathbf{x}-\mathbf{y})+\frac{\partial^{2}}{\partial x_{i} \partial y_{j}} \frac{1}{4 \pi|\mathbf{x}-\mathbf{y}|} \quad(i, j=1,2,3) \\
\left\{\pi_{\mu}(\mathbf{x}, t), \pi_{\nu}(\mathbf{y}, t)\right\}^{*}=\left\{A_{\mu}(\mathbf{x}, t), A_{\nu}(\mathbf{y}, t)\right\}^{*}=0 \quad(\mu, \nu=1,2,3,4)
\end{gathered}
$$

Taking into consideration the Fourier transformation of the Coulomb potential, Eq. (31) takes the form

$$
\left\{\pi_{i}(\mathbf{k}), A_{j}(\mathbf{q})\right\}^{*}=-(2 \pi)^{3} \delta(\mathbf{k}+\mathbf{q})\left(\delta_{i j}-\frac{k_{i} k_{j}}{\mathbf{k}^{2}}\right) .
$$

The projection operator in the right side of Eq. (33) extracts the physical transverse components of vectors. 
Following the general method, we can set all second class constraints strongly to zero, and as a result, only transverse components of the vector potential $A_{\mu}$ and momentum $\pi_{\mu}$ are physical independent variables. Pairs of operators $(28)$ are absent in the reduced physical phase space and the physical Hamiltonian becomes $H^{p h}=\int d^{3} x \mathcal{E}$. Equations of motion obtained from this Hamiltonian coincide with Eqs. (23)-(25) at $u(x)=v(x)=0$ (the Poisson brackets are replaced by the Dirac brackets). In the quantum theory, we should substitute Dirac's brackets by the quantum commutators according to the prescription $\{., .\}^{*} \rightarrow-i[.,$.$] . The fields \mathbf{E}, \mathbf{B}, \mathbf{D}, \mathbf{H}$ are invariants of the gauge transformations and are measurable quantities (observables).

\section{Conclusion}

We have just considered the effective non-linear electrodynamics, which is equivalent to Maxwell's theory on NC spaces due to the Seiberg-Witten map. The canonical conservative, symmetric non-conservative energy-momentum tensors, and their non-zero traces were found. We have shown that there is a trace anomaly which is related with the violation of the dilation symmetry at the classical level. The trace anomaly is absent in the case of the plane electromagnetic waves. At high energy (on short distances), when spacetime might be non-commutative, the trace anomaly can contribute to the cosmological constant, and as a result, effect cosmology and physics of early universe.

Dirac's quantization of the effective non-linear electromagnetic theory is similar to the quantization of classical electrodynamics, and includes first class constraints. The gauge fixing approach, on the basis of Coulomb's gauge, leads to second class constraints and transition from the Poisson brackets to the Dirac brackets. For consideration of the vacuum state one needs to construct the Fock representation or the wave functionals. The normalization conditions are formulated here in terms of functional integrals with the corresponding gauge conditions. This may involve, however, the introducing ghosts and negative norm states, which is beyond the Dirac approach. The gauge covariant Dirac quantization does not violate the Lorentz invariance and locality in space, and has an advantage compared to the reduced phase space approach (see [17]).

The quantization of the Maxwell theory on NC spaces within BRSTscheme was investigated in [4], [0], [19]. 


\section{References}

[1] Seiberg, N., and Witten, E., JHEP 9909, 032 (1999); arXiv:hepth/9908142.

[2] Snyder, H., Phys. Rev. 71, 38-41 (1947); 72, 68-72 (1947).

[3] Connes, A., Noncommutative Geometry, Academic Press, 1994.

[4] Bichl, A., Grimstrup, J., Popp, L., Schweda, M., and Wulkenhaar, R., arXiv:hep-th/0102044.

[5] Fruhwirth, I., Grimstrup, J.M., Morsli, Z., Popp, L., Schweda, M., arXiv:hep-th/0202092.

[6] Jackiw, R., Nucl. Phys. Proc. Suppl. 108, 30-36 (2002); arXiv:hepth/0110057.

[7] Guralnic, Z., Jackiw, R., Pi, S.Y., and Polychronakos, A.P., Phys. Lett. B 517, 450-456 (2001); arXiv:hep-th/0106044.

[8] Gomis, J., and Mehen, T., Nucl. Phys. B591, 265-276 (2000); arXiv:hepth/0005129.

[9] Aharony, O., Gomis, J., and Mehen, T., JHEP 0009, 023 (2000); arXiv:hep-th/0006236.

[10] Riad, I.F., and Sheikh-Jabbari, M.M., JHEP 0008, 045 (2000); arXiv:hep-th/0008132.

[11] Sheikh-Jabbari, M.M., Phys. Rev. Lett. 84, 5265-5268 (2000); arXiv:hep-th/0001167.

[12] Hayakawa, M., Phys. Lett. B478, 394-400 (2000); arXiv:hepth/9912094; arXiv:hep-th/9912167.

[13] Kruglov, S.I., Annales Fond. Broglie (in press); arXiv:hep-th/0110059.

[14] Landau, L.D. and Lifshits, E.M., The Classical Theory of Fields, Pergamon Press, 1975.

[15] Dirac, P.A.M., Lectures on Quantum Mechanics, Yeshiva University, New York, 1964. 
[16] Hanson, A., Regge, T., Teitelboim, C., Constrained Hamiltonian Systems, Accademia Nationale Dei Lincei, Roma, 1976.

[17] Henneaux, M., and Teitelboim, C., Quantization of Gauge Systems, Princeton University Press, Princeton, New Jesey, 1992.

[18] Matschull, H.J., arXiv:quant-ph/9606031.

[19] Wulkenhaar, R., JHEP 0203, 024 (2002); arXiv:hep-th/0112248. 